\title{
Two designs of the S4.BEN01 magnet for the CBETA splitter/merger
}

\author{
N. Tsoupas, S. Berg, F. Meot, V. Ptitsyn, \\ D. Trbojevic, J. Tuozzolo
}

\section{Collider-Accelerator Department Brookhaven National Laboratory Upton, NY 11973}

\author{
U.S. Department of Energy \\ Office of Science, Office of Nuclear Physics
}




\title{
Two designs of the S4.BEN01 Magnet for the CBETA Splitter/Merger
}

\author{
N. Tsoupas, S. Berg, F. Meot, V. Ptitsyn, D. Trbojevic, J. Tuozzolo
}

\begin{abstract}
The splitter/merger section of the CBETA project [1] consists of 4 beam lines as shown in Fig. 1. Two of the functions of the splitter's/merger's lines is to match the beam parameters at the exit of the Energy Recovery Linac (ERL) to the beam parameters at the entrance of the Fixed Field Alternating Gradient (FFAG) arc, and also place the reference particles of the beam bunches at the entrance of the FFAG arc on specified trajectories according to their energies. In this technical note we are presenting results from the 2D and 3D electromagnetic analysis of the S4.BEN01 magnet which is one of the dipole magnets of the $150 \mathrm{MeV}$ line of the splitter/merger. In particular we present results from two designs of the S4.BEN01 magnet, one based on iron dominated current-excited magnet, and the other design based on Halbach-type permanent magnet. An evaluation of the two designs will be given in the section under "conclusion".
\end{abstract}

\section{Introduction}

Figure 1 is a schematic diagram of the CBETA splitter showing the four beam lines and the S4.BEN01 magnet which is the subject of this study.

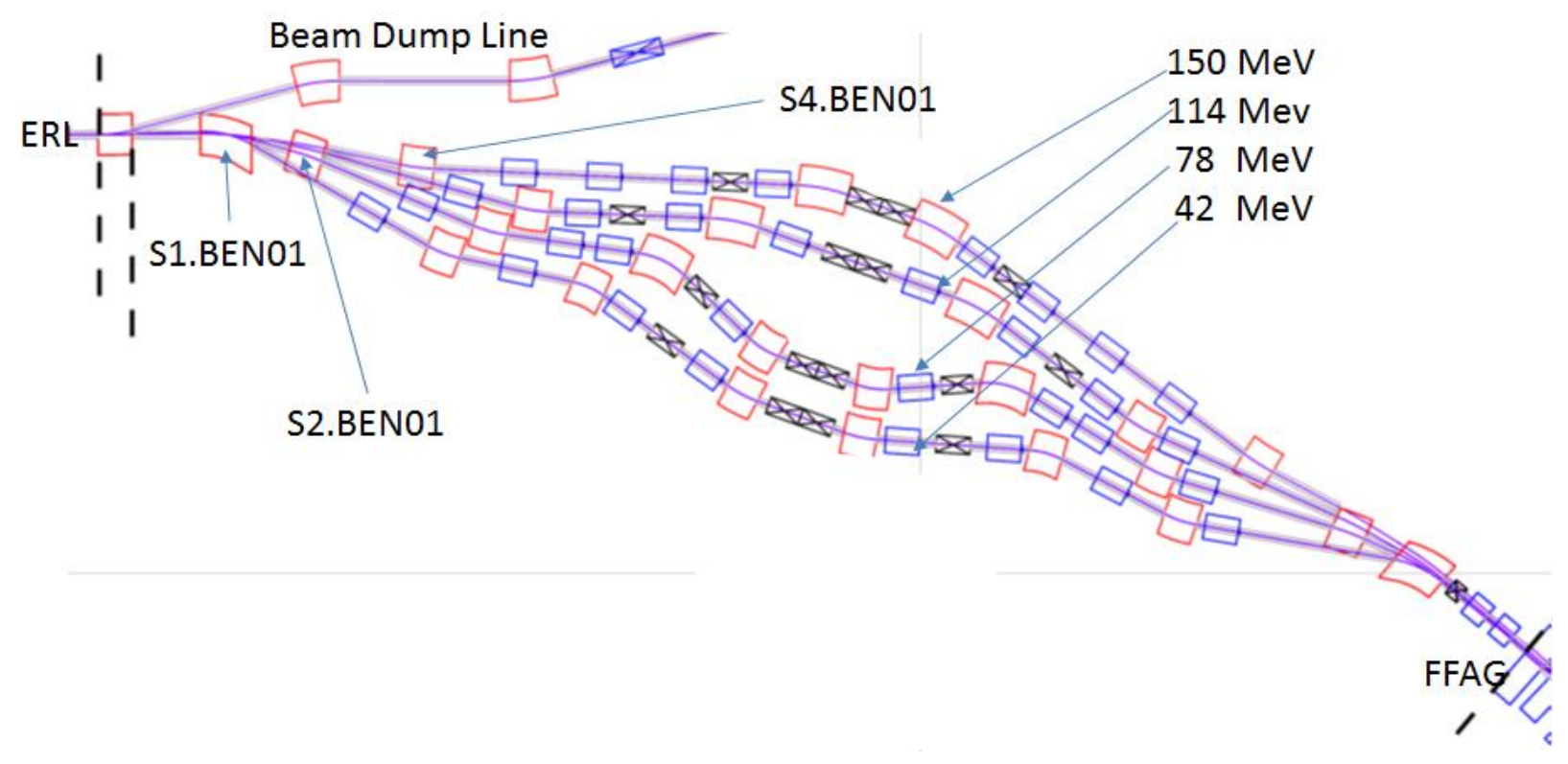

Figure 1. The four beam lines of the SBETA splitter. The150 MeV lines's S4.BEN01-magnet which is the study of this technical note is shown in the figure.

The design of the magnet depends partly on the beam trajectories of the two reference particles, namely the $114 \mathrm{MeV}$ and $150 \mathrm{MeV}$, at the region of the S4.BEN01 magnet. These trajectories have been obtained 
from the file "cbeta_4pass_20161117.global_orbit" and are shown in fig. 2 as the two colored traces which are labelled with the values of the beam energies. The coordinates of the reference particles at the entrance and exit of magnet are at the center of the green circles in fig. 2 and their numerical values of the coordinates appear in the last six columns of Table 1 . The center of the green circles on the figure represent the reference particles of the 114 , and $150 \mathrm{MeV}$ bunches and the reference particle of the two electron beams should be at least $1.2 \mathrm{~cm}$ away from any material. The red areas represent the coils of the magnet. The walls of the vacuum chamber are the thin orange stripes, and the light-green thin quadrilateral region is the coil-retaining nonmagnetic sheet. The yellow quadrilateral region is the vacuum pipe of the $114 \mathrm{MeV}$ beam. The vacuum pipe should be made of magnetic material.

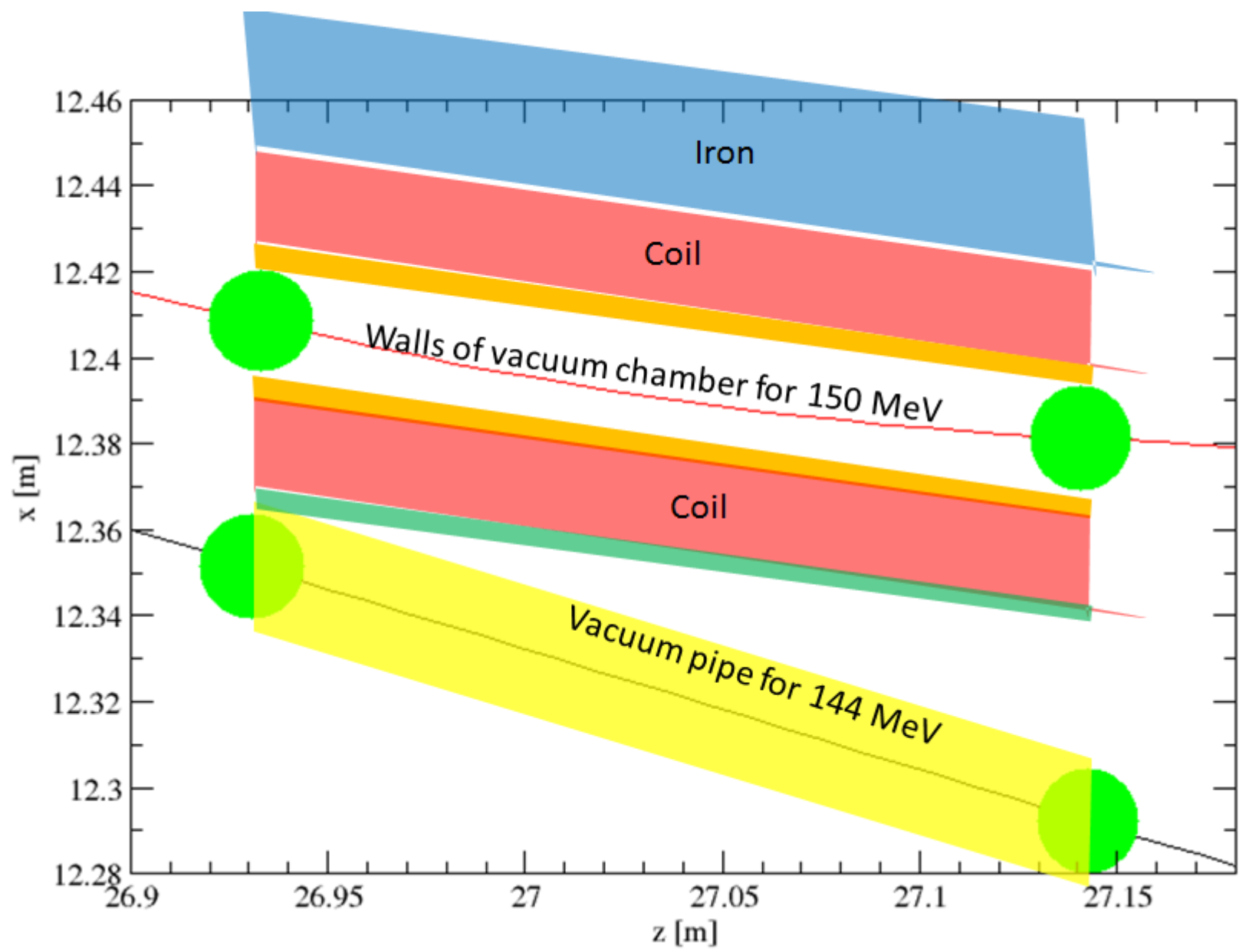

Figure2. The schematic diagram of the cross section of the median plane of the S4.BEN01 magnet. The thin traces on the figure are the trajectories of the reference particles of the two electron bunches on the median plane at the region of the S4.BEN01 magnet. The center of the green circles on the figure represent the reference particles of the 114 , and $150 \mathrm{MeV}$ bunches at the entrance and exit of the magnet. The red areas represent the coil of the magnet. The walls of the vacuum chamber are the thin orange stripes, and the light-green stripe is the coil-retaining nonmagnetic sheet. The yellow quadrilateral region is the vacuum pipe of the $114 \mathrm{MeV}$ beam. 
Table 1. The kinetic energy of the 114 , and $150 \mathrm{MeV}$ electron bunches and the angle of bend in the 150 $\mathrm{MeV}$ bunch in the S4.BEN01 magnet. The coordinates of the reference particles of the 114, and $150 \mathrm{MeV}$ bunches at the entrance and exit of the magnet appear in the last six columns.

\begin{tabular}{|c|c|c|c|c|c|c|c|c|c|}
\hline \multicolumn{3}{|c|}{} & \multicolumn{3}{|c|}{ Entrance } & \multicolumn{3}{|c|}{ Exit } \\
\hline $\mathrm{KE}[\mathrm{GeV}]$ & gamma & $\mathrm{P}[\mathrm{GeV} / \mathrm{c}]$ & $\begin{array}{c}\text { Ang. Bend } \\
{[\mathrm{deg}]}\end{array}$ & $\mathrm{X}_{\text {in }}[\mathrm{m}]$ & $\mathrm{x}_{\text {in }}[\mathrm{deg}]$ & $\mathrm{z}_{\text {in }}[\mathrm{m}]$ & $\mathrm{X}_{\text {out }}[\mathrm{m}]$ & $\mathrm{x}_{\text {out }}[\mathrm{deg}]$ & $\mathrm{Z}_{\text {out }}[\mathrm{m}]$ \\
\hline 0.114 & 224.092 & 0.1145 & 0.0 & 12.35158 & -15.6578 & 26.9309 & 12.29221 & -15.6578 & 27.14276 \\
\hline 0.150 & 294.542 & 0.1505 & 9.30225 & 12.40842 & -11.8948 & 26.9330 & 12.38121 & -2.59254 & 27.14101 \\
\hline
\end{tabular}

The strength of the $0.2 \mathrm{~m}$ long S4.BEN01 magnet which is 0.406167 [T] and the angle of bend of the 150 $\mathrm{MeV}$ high energy bunch appears in column four of Table 1.

\section{The S4.BEN01 Magnet Specifications}

The schematic diagram of the S4.BEN01 magnet's cross section on the median plane is shown in fig. 2. Only the $150 \mathrm{MeV}$ high energy bunch is bend by the magnet.

The yellow stripe in fig. 2, around the $114 \mathrm{MeV}$ trajectory is the $0.2 \mathrm{~cm}$ thick vacuum pipe which is made with magnetic material and is copper platted inside.

The thin orange stripes are the walls of the $0.2 \mathrm{~cm}$ thick vacuum chamber, and the green stripe is the 0.2 mm thick nonmagnetic coil-retaining plate.

The vacuum pipe of the $114 \mathrm{MeV}$ beam bunch is made of magnetic material to act as a magnetic shield for the $144 \mathrm{MeV}$ beam bunch.

From the values of the coordinates in Table 1 the distance of the reference particles of the $144 \mathrm{MeV}$ and the $150 \mathrm{MeV}$ beam bunches at the entrance of the magnet is $5.68 \mathrm{~cm}$ therefore the magnet should be a septum like magnet.

The central particles of each of the four bunches should be at least $1.2 \mathrm{~cm}$ away from any material. This constrain is taken into account in the design of the magnet.

Table 2 contains the specifications of the S4.BEN01 septum magnet.

Table 2. S4.BEN01 Magnet specifications

\begin{tabular}{|c|c|c|c|c|c|c|c|c|}
\hline $\begin{array}{c}\text { Length } \\
{[\mathrm{m}]}\end{array}$ & $\begin{array}{c}\mathrm{B}_{\text {field }} \\
{[\mathrm{T}]}\end{array}$ & $\begin{array}{c}\text { Bend } \\
\text { angle }\end{array}$ & $\begin{array}{c}\text { Sagitta } \\
{[\mathrm{cm}]}\end{array}$ & $\begin{array}{c}\text { Gap } \\
{[\mathrm{cm}]}\end{array}$ & $\begin{array}{c}\text { Septum } \\
{[\mathrm{cm}]}\end{array}$ & $\begin{array}{c}\text { Hor. Aper. } \\
{[\mathrm{cm}]}\end{array}$ & $\begin{array}{c}\text { Current } \\
{[\text { A.turns }]}\end{array}$ & $\begin{array}{c}\mathrm{J} \\
{\left[\mathrm{A} / \mathrm{cm}^{2}\right]}\end{array}$ \\
\hline 0.2 & 0.40617 & $9.3^{\circ}$ & 0.2 & 3.4 & 2.68 & 3.5 & 10990 & 1400 \\
\hline & & & & & & & & \\
\hline
\end{tabular}

In this technical note we define as "septum-thickness" the available transverse space for the copper coil. The septum-thickness which the available space to place the conductors of the magnet's coil is calculated in the following way.

septum-thickness=

=(central particles spacing between 144 and $150 \mathrm{MeV}$ bunches)-2x(distance_of_refer_paticle_from_chamber_wall)$3 \mathrm{x}$ (chamber_thickness) $=5.68 \mathrm{~cm}-2 \times 1.2 \mathrm{~cm}-3 \times 0.2 \mathrm{~cm}=2.68 \mathrm{~cm}$.

\section{D Design of the electromagnet}

Fig. 3 is a cross section of the septum magnet. The blue circular pipe on fig. 3 is the $144 \mathrm{MeV}$ vacuum pipe which should be of magnetic material to shield magnetically the $144 \mathrm{MeV}$ electron bunch. The 
region with the blue color is the iron of the magnet, and the red-colored regions are the conductors of the coil. In this study we assume 8 conductors per coil.

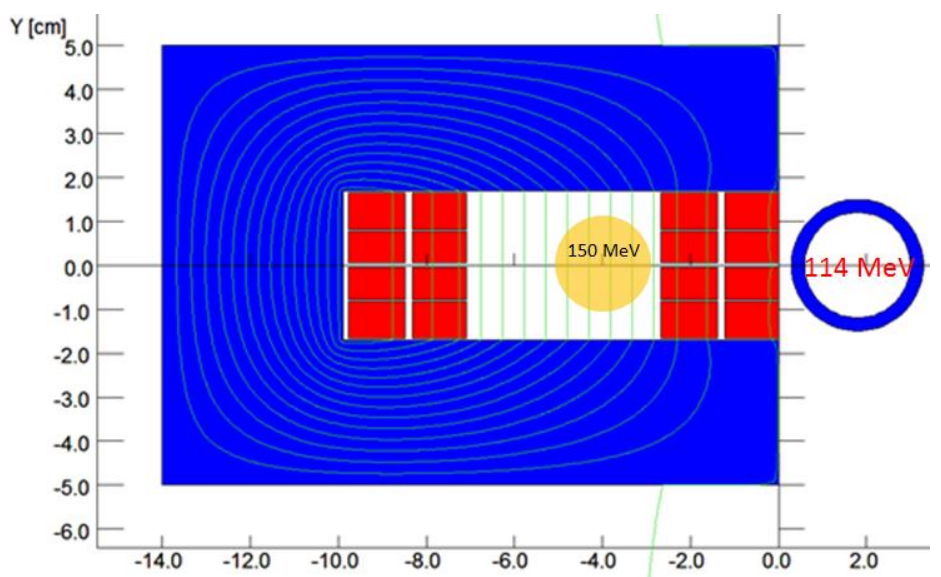

Figure 3. The cross section of the S4.BEN01 septum magnet. The blue-colored regions represent magnetic iron, and the red-colored regions are the conductors of the coil. In this study we assume 8 conductors for the coil.

The B-field in the magnet is $0.40617 \mathrm{~T}$ with a current density of $1400 \mathrm{~A} / \mathrm{cm}^{2}$. The field homogeneity in the region were the three beams are circulating is shown in Fig. 4.

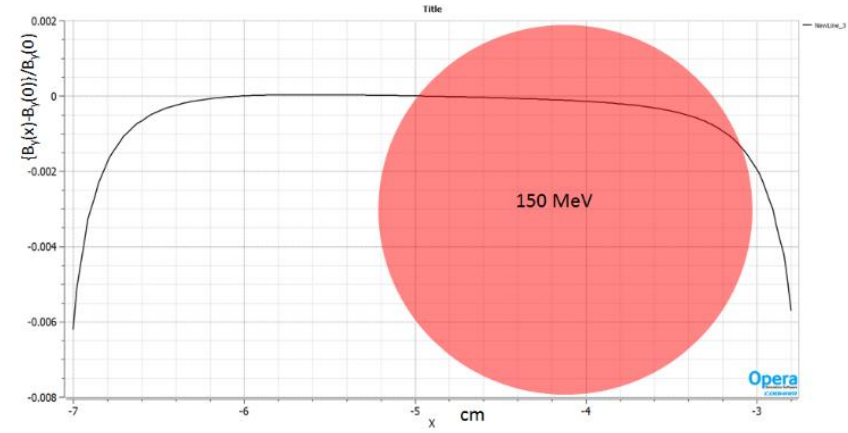

Figure 4. The field homogeneity for the $150 \mathrm{MeV}$ beam.

Fig. 5 shows that the $3 \mathrm{~cm}$ thick iron yoke is sufficient to keep the iron below saturation.

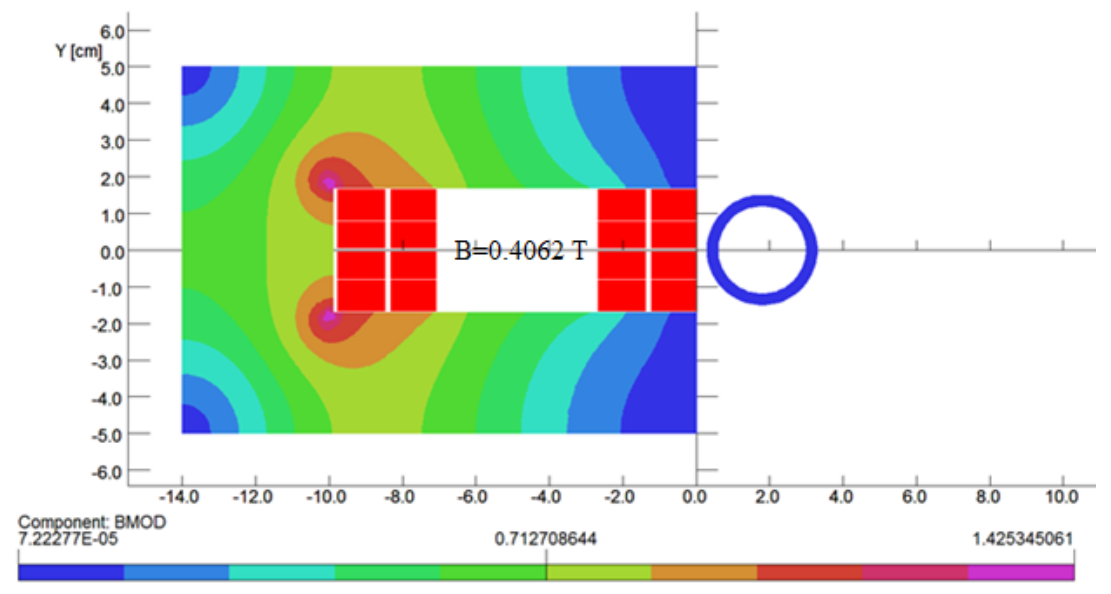

Figure 5. The maximum modulus of the magnetic field of $1.42 \mathrm{~T}$ of the iron yoke indicates that the magnet runs below saturation. 
The vacuum pipe of the $150 \mathrm{MeV}$ beam is made of magnetic iron and serves to shield the beam from the magnetic field generated by the magnet. Fig. 6 shows that the magnetic field inside the vacuum pipe is less than $9 \times 10^{-7} \mathrm{~T}$.

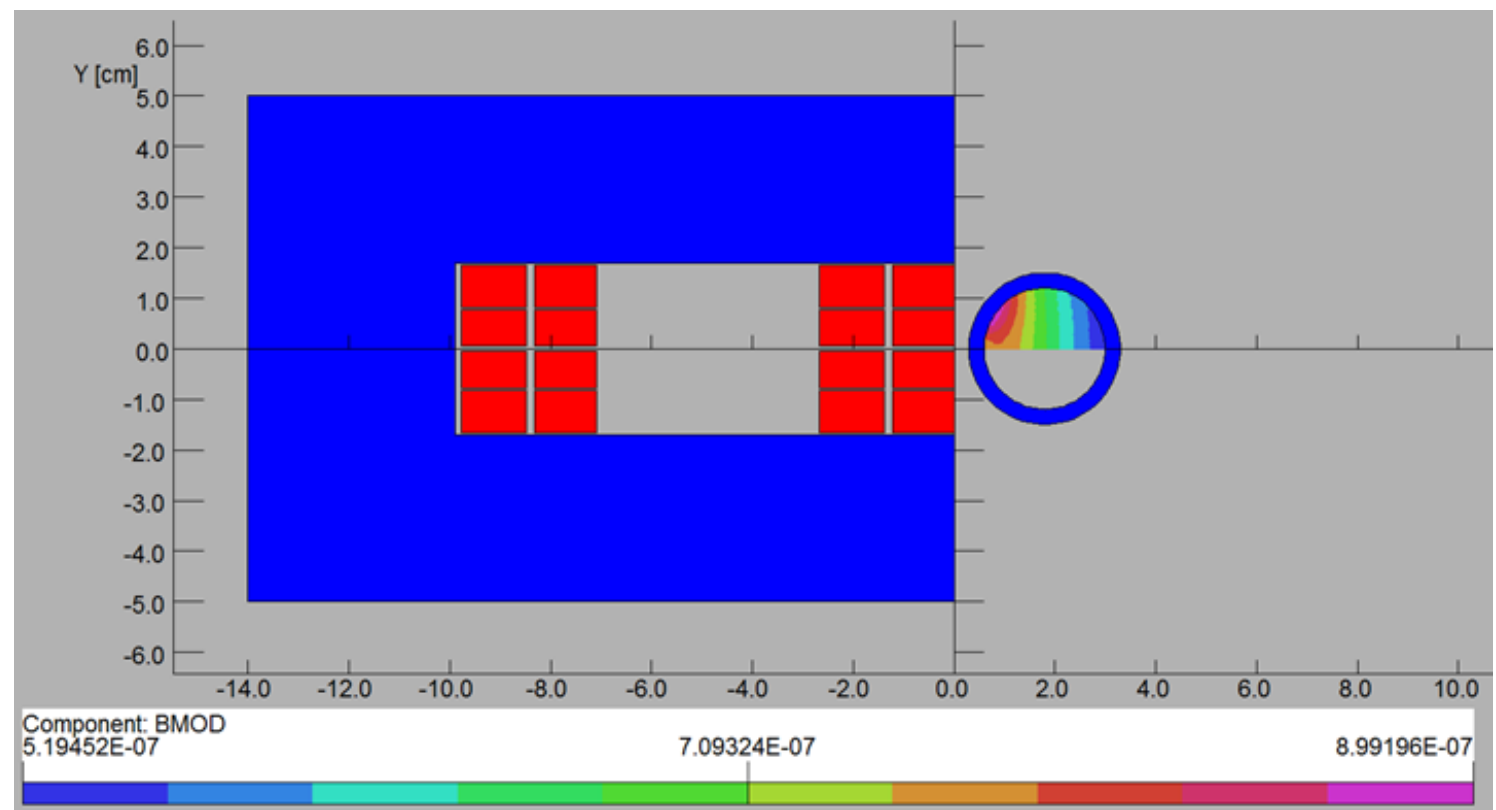

Figure 6. The magnetic field inside the vacuum pipe of the $144 \mathrm{MeV}$ beam is less than $9 \times 10^{-7} \mathrm{~T}$. The material of the vacuum pipe is of magnetic iron.

If the vacuum pipe is of nonmagnetic material the magnetic field inside the pipe is $\sim 9$ Gauss as shown in Fig. 7.

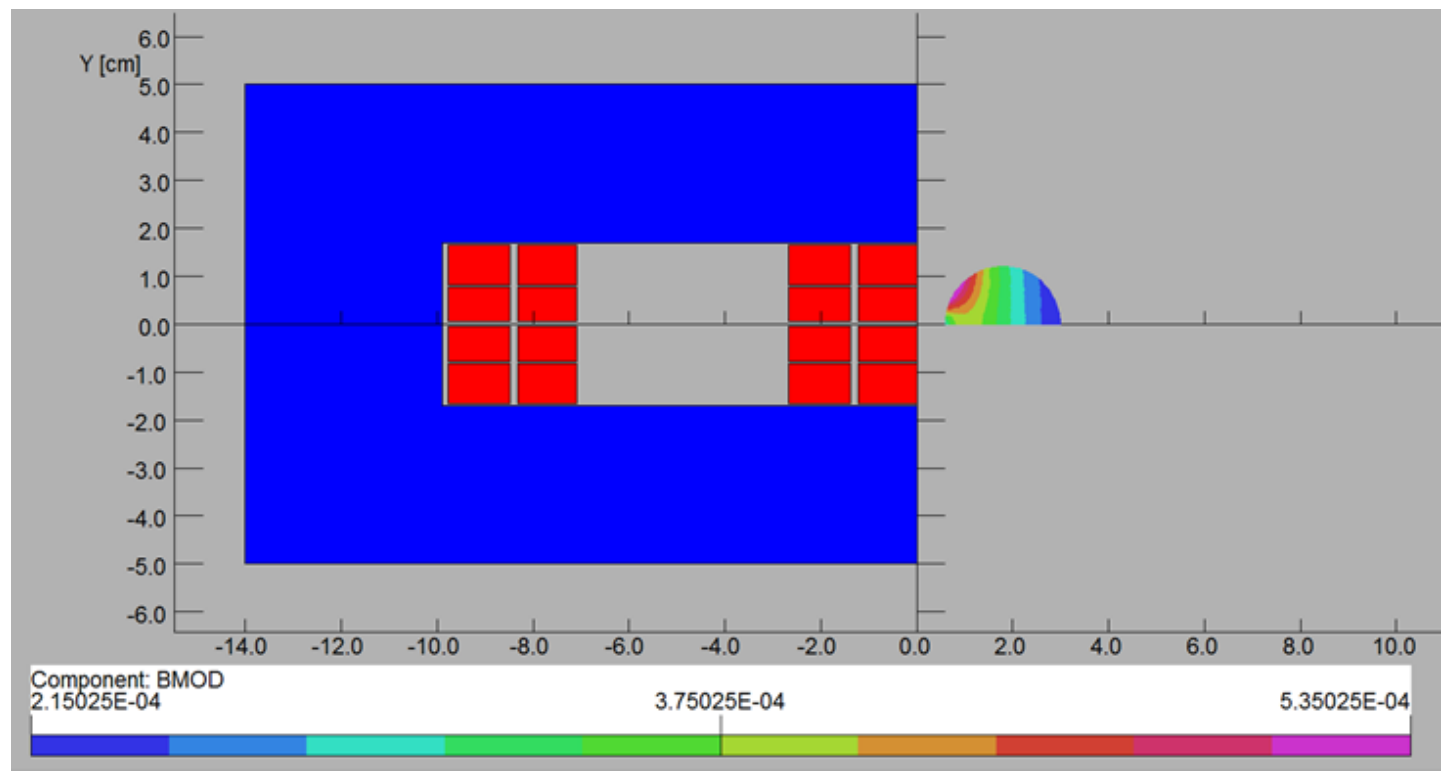

Figure 7. The magnetic field inside a non-magnetic vacuum pipe of the $144 \mathrm{MeV}$ beam pipe is $\sim 5.3$ Gauss. 
We therefore recommend that the vacuum pipe is make of magnetic material to minimize the effect of the septum magnet on the $42 \mathrm{MeV}$ beam.

\section{D Design}

The upper left picture of Fig. 8 is an isometric view of the magnet's model generated with the preprocessor of the OPERA computer code. The cylindrical pipe on the right side of the magnet is the vacuum pipe for the $144 \mathrm{MeV}$ beam and is made of magnetic material to minimize the magnetic field of the magnet along the trajectory of the $144 \mathrm{MeV}$ beam. The plot on the right bottom of the picture is a graph with two plots of the magnetic field along the center of the vacuum pipe. One of the plots corresponds to the field with the short pipe (upper left corner) and the other plot to that of the magnet with the long pipe (upper right corner).

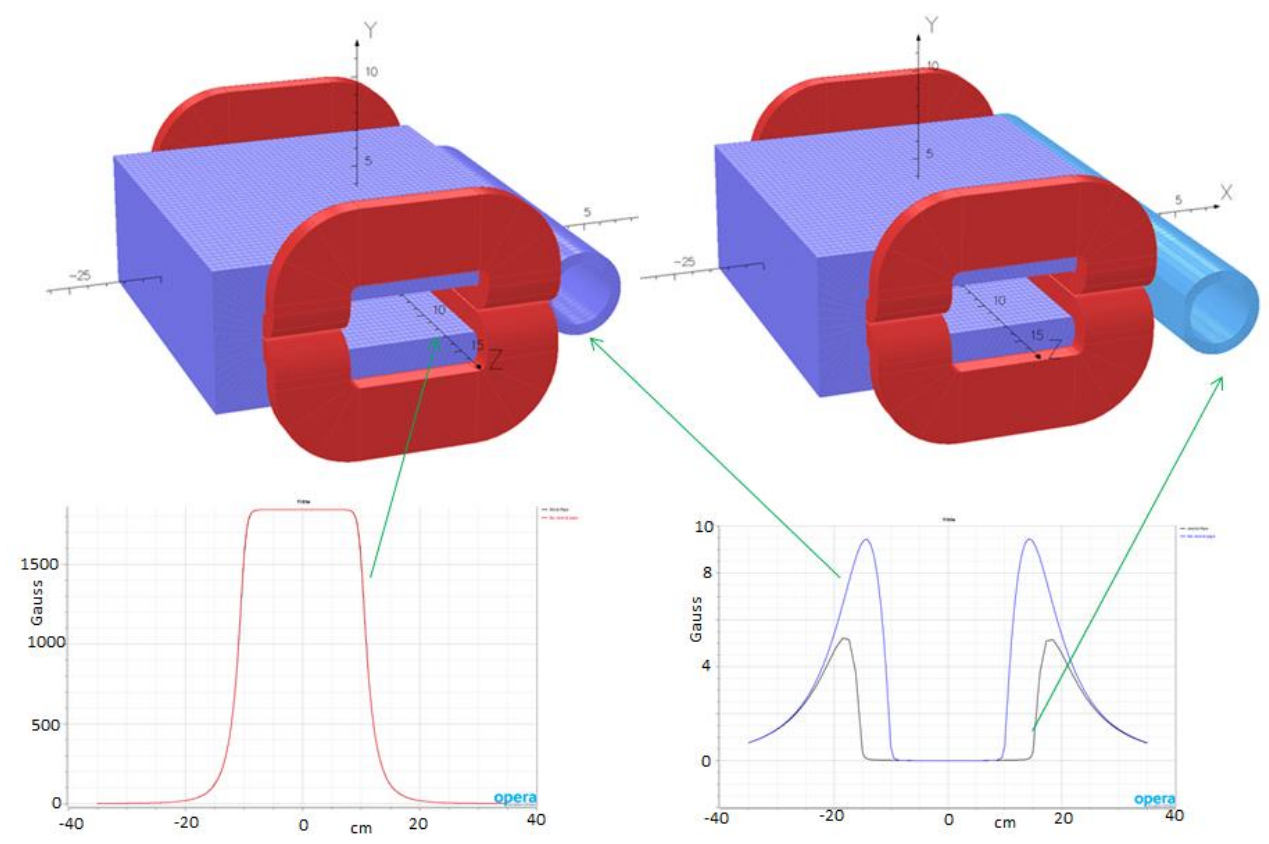

Figure 8. a) (Upper left) isometric view of the S4.BEN01 septum magnet, with the magnetic pipe for the $144 \mathrm{MeV}$ beam. b) (Upper right) the same magnet as in (a) but with a longer vacuum pipe for better magnetic shielding of the $144 \mathrm{MeV}$ beam. c) (Bottom left) The magnetic field inside the magnet as a function along the z-axis. d) (Bottom right) The magnetic field along the $144 \mathrm{MeV}$ trajectory for the sort and the long magnetic pipes respectively.

\section{Halbach type magnet as an alternative for the S4.BEN01 electromagnet}

In this section we present the results of the study on a Halbach type dipole magnet made of permanent magnet material to replace the S4.BEN01 electromagnet discussed earlier. A comparison between the electromagnet and the Halbach type dipole magnet will be given in the "conclusion" section.

\section{Geometric consideration}

Since the permanent magnet can provide stronger fields $(\sim 0.67 \mathrm{~T})$ as compared to the field of the electromagnet $(0.406 \mathrm{~T})$, we can use a $12 \mathrm{~cm}$ long Halbach magnet instead of $20 \mathrm{~cm}$ long electromagnet, 
and we place the longitudinal center of the Halbach magnet at the same location as the electromagnet's center to provide the same angle of bends and trajectory for the $150 \mathrm{MeV}$ electron bunch as for the electromagnet. Fig. 9 shows a schematic diagram of the cross section at the median plane of the Halbach type of magnet. The center of the blue circles represent the global coordinates of the reference particles of the 144 , and $150 \mathrm{MeV}$ bunches at the entrance and exit of the $12 \mathrm{~cm}$ long Halbach magnet. Table 3 provides the coordinates of the reference particles of these bunches at the entrance and exit of the magnet. The orange stripes are the $0.2 \mathrm{~cm}$ thick walls of the vacuum chamber for the $150 \mathrm{MeV}$ beam, and the green stripes is the retaining material of the permanent magnet material shown in red. The retaining material of the permanent magnets could be of magnetic material. The yellow quadrilateral shape is the $0.2 \mathrm{~mm}$ thick vacuum pipe of the $144 \mathrm{MeV}$ beam. The pipe is made of magnetic material.

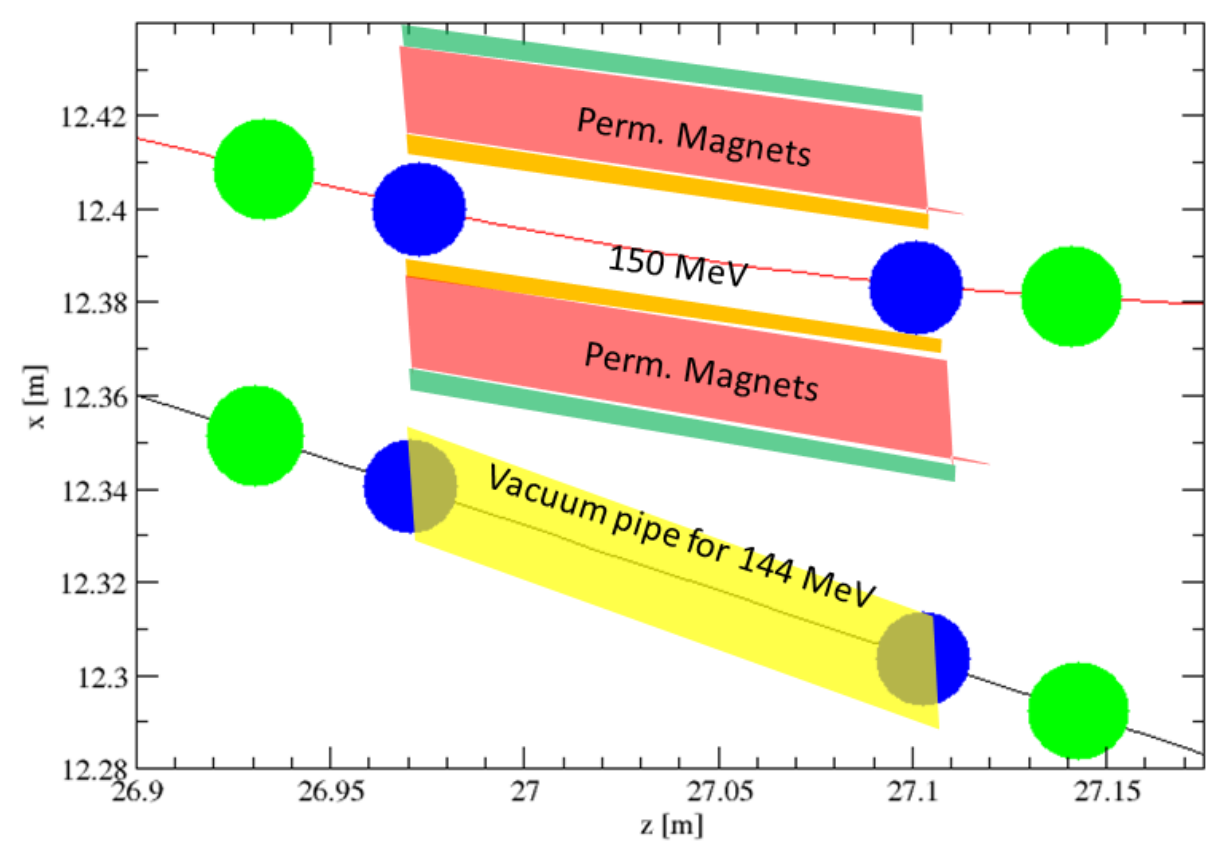

Figure 9. Schematic diagram of the cross section on the median plane of the $12 \mathrm{~cm}$ long Halbach magnet. The thin traces are the trajectories of the reference particles of the 144 and $150 \mathrm{MeV}$ electron bunches on the median plane at the region of the S4.BEN01 magnet. The center of the blue circles on the figure represent the reference particles of the two bunches at the entrance and exit of the $12 \mathrm{~cm}$ long Halbach type dipole magnet.

Table 3. The kinetic energy of the two electron bunches and their angles of bend in the S4.BEN01 magnet. The coordinates of the reference particles of the two bunches at the entrance and exit of the Halbach magnet appear in the last six columns.

\begin{tabular}{|c|c|c|c|c|c|c|c|c|c|}
\hline \multicolumn{4}{|c|}{} & \multicolumn{3}{|c|}{ Entrance } & \multicolumn{3}{|c|}{ Exit } \\
\hline $\mathrm{KE}[\mathrm{GeV}]$ & gamma & $\mathrm{P}[\mathrm{GeV} / \mathrm{c}]$ & $\begin{array}{c}\text { Ang. Bend } \\
{[\mathrm{deg}]}\end{array}$ & $\mathrm{X}_{\text {in }}[\mathrm{m}]$ & $\mathrm{x}_{\text {in }}^{\prime}[\mathrm{deg}]$ & $\mathrm{Z}_{\text {in }}[\mathrm{m}]$ & $\mathrm{X}_{\text {out }}[\mathrm{m}]$ & $\mathrm{x}_{\text {out }}[\mathrm{deg}]$ & $\mathrm{Z}_{\text {out }}[\mathrm{m}]$ \\
\hline 0.114 & 224.092 & 0.1145 & 0.0 & 12.34037 & -15.6578 & 26.9709 & 12.30342 & -15.6578 & 27.10276 \\
\hline 0.150 & 294.542 & 0.1505 & 9.30225 & 12.40000 & -11.8948 & 26.9730 & 12.38302 & -2.59254 & 27.10101 \\
\hline
\end{tabular}


From the values of the coordinates shown in Table 3 we conclude that the distance between the central particles of the $144 \mathrm{MeV}$ and the $150 \mathrm{MeV}$ at the entrance of magnet is $5.96 \mathrm{~cm}$ and their distance at the exit of the magnet is $7.96 \mathrm{~cm}$. Given the constrain that the center of the beam bunch should be $1.2 \mathrm{~cm}$ from the inner surface of the vacuum chamber whose thickness can be $\sim 2 \mathrm{~mm}$, the available space for permanent magnet material is $=5.96 \mathrm{~cm}-2 \times 1.2 \mathrm{~cm}-3 \times 0.2 \mathrm{~cm}=2.96 \mathrm{~cm}$. This distance of $2.96 \mathrm{~cm} \mathrm{can}$ accommodate the permanent magnet material and also material to maintain the temperature of the permanent magnet constant.

\section{D Design of the $12 \mathrm{~cm}$ long Halbach type magnet}

In this section we present the 2D electromagnetic analysis of an alternative design of the S4.BEN01 electromagnet. This design is based on a Halbach type of magnet. Fig. 10 shows the cross section of such Halbach type dipole magnet made of 16 permanent magnet wedges. The inner and outer radii of the Halbach annulus are 2.0 and $3.5 \mathrm{~cm}$ respectively and the magnet generates a field of $0.65 \mathrm{~T}$. The inner radius of the Halbach magnet can easily accommodate a $2 \mathrm{~mm}$ thick vacuum chamber and satisfy the constrain for the reference trajectory of the electron bunch to be $1.2 \mathrm{~cm}$ away from the vacuum wall.

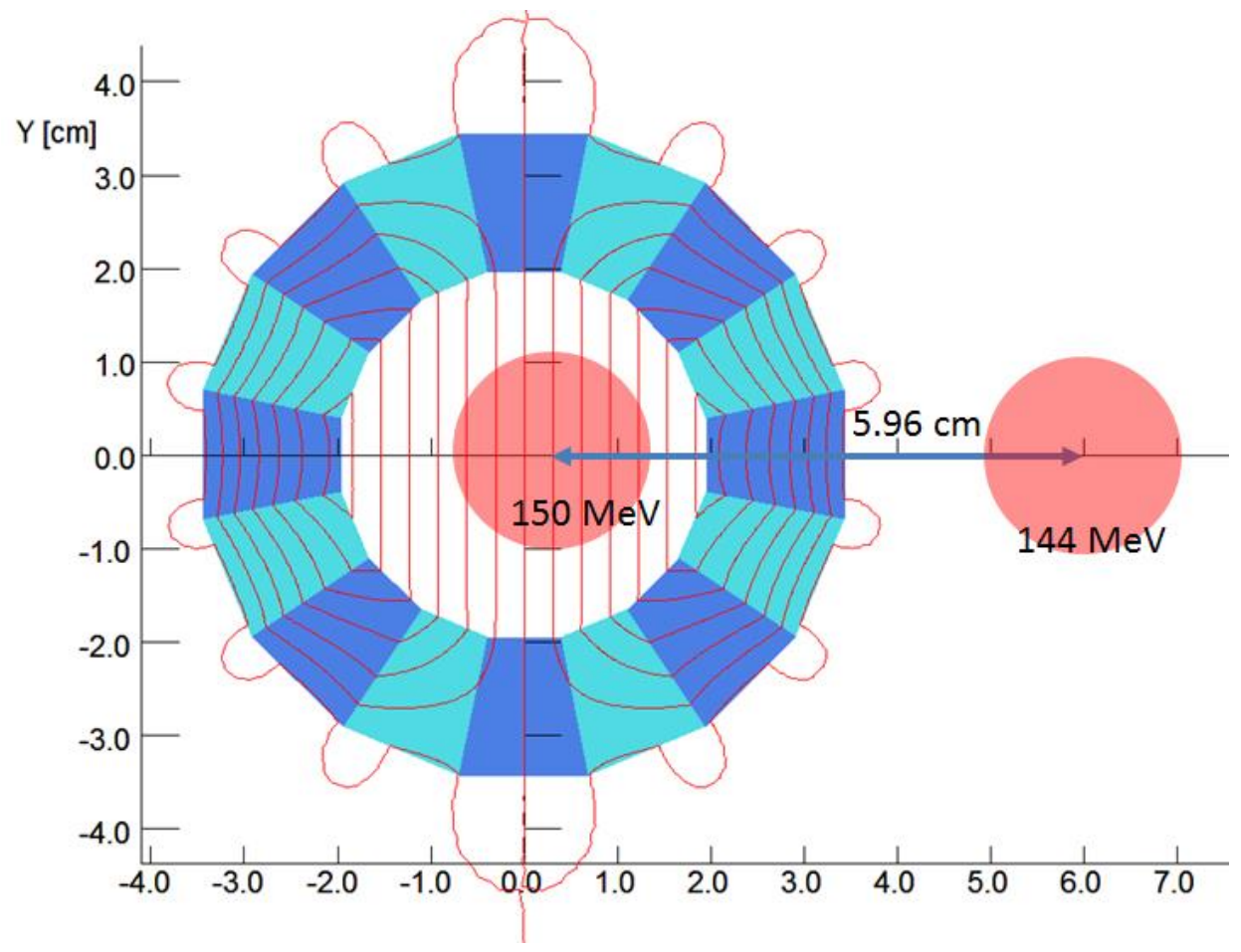

Figure 10. A Halbach type dipole magnet made of 16 permanent magnet wedges. The permanent magnet material used in the design is NdFeB_N35EH and the dipole field generated is $0.65 \mathrm{~T}$. The red traces on the figure are the equipotential vector lines. The centers of the red circles which are at a distance of 5.96 $\mathrm{cm}$, are the reference particles for the 144 and $150 \mathrm{MeV}$ electron bunches.

The permanent magnet material used in the design of the magnet is NdFeB_N35EH and the dipole field generated by the magnet is $0.65 \mathrm{~T}$. The red traces on the figure are the equipotential vector lines. The field uniformity within $\mathrm{x}= \pm 1.5 \mathrm{~cm}$ is better than $1.0 \mathrm{x} 10^{-3}$ and is shown in Fig. 11 . The reason we have set the field of the magnet at the value of $\sim 0.65 \mathrm{~T}$ is that our manufacturer for permanent magnets supplies 
wedges of $6 \mathrm{~cm}$ long, and the integrated field of the $0.65 \mathrm{~T}$ and 2x6 cm long Halbach magnet generates the same integrated dipole field as the $20 \mathrm{~cm}$ long electromagnet we discussed earlier.

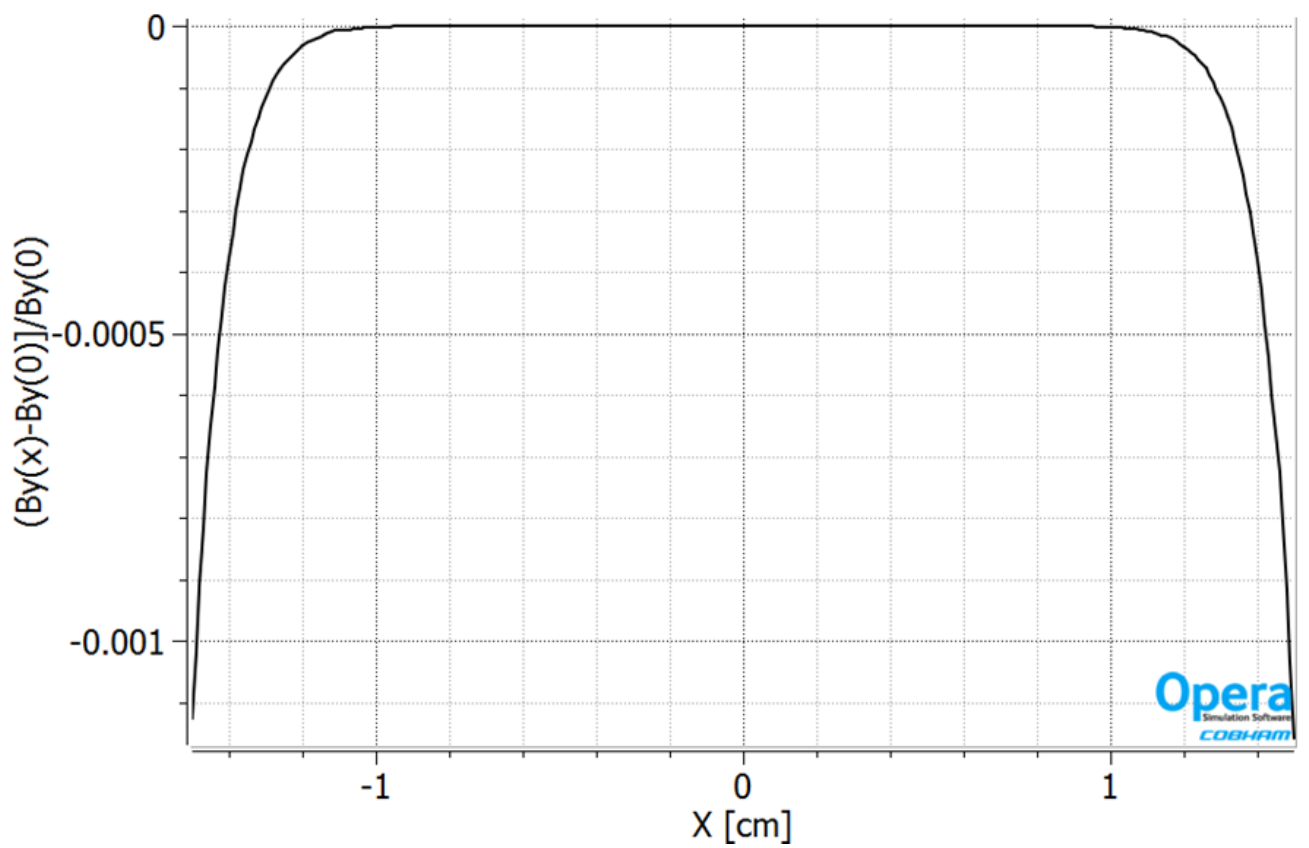

Figure 11. The dipole field homogeneity of the Halbach magnet in a range $\mathrm{x}= \pm 1.5 \mathrm{~cm}$ is less than $1 \times 10^{-3}$. 3D Design of the $12 \mathrm{~cm}$ long Halbach type magnet

In this section we study the 3D design of the $12 \mathrm{~cm}$ long Halbach magnet. Figure 12 is an isometric view of the Halbach magnet which can generate the same integrated field as the S4.BEN01 electromagnet. The inner and outer radii are 2.0 and $3.5 \mathrm{~cm}$ respectively. The field homogeneity at the center of the magnet is shown in Fig. 13, and is better than $1 \times 10^{-3}$.

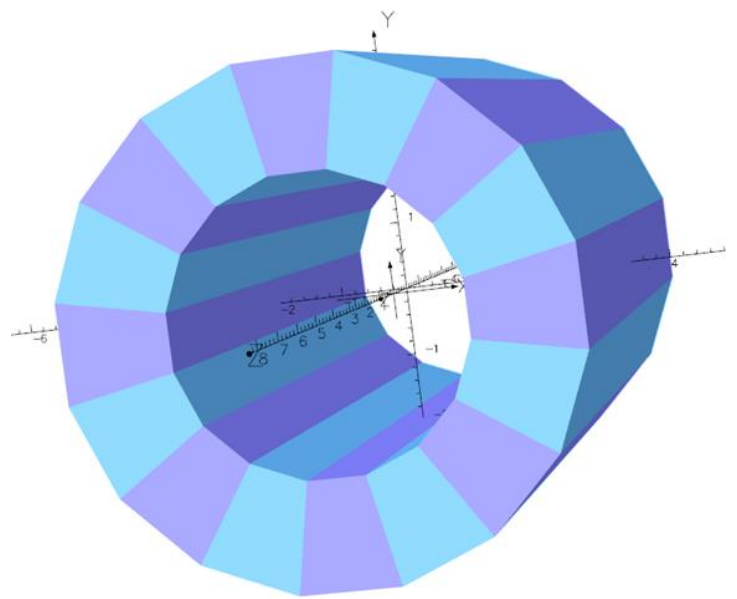

Figure 12. Isometric view of the $12 \mathrm{~cm}$ long Halbach magnet which can generate the same integrate dipole field as the S4.BEN01 electromagnet. 


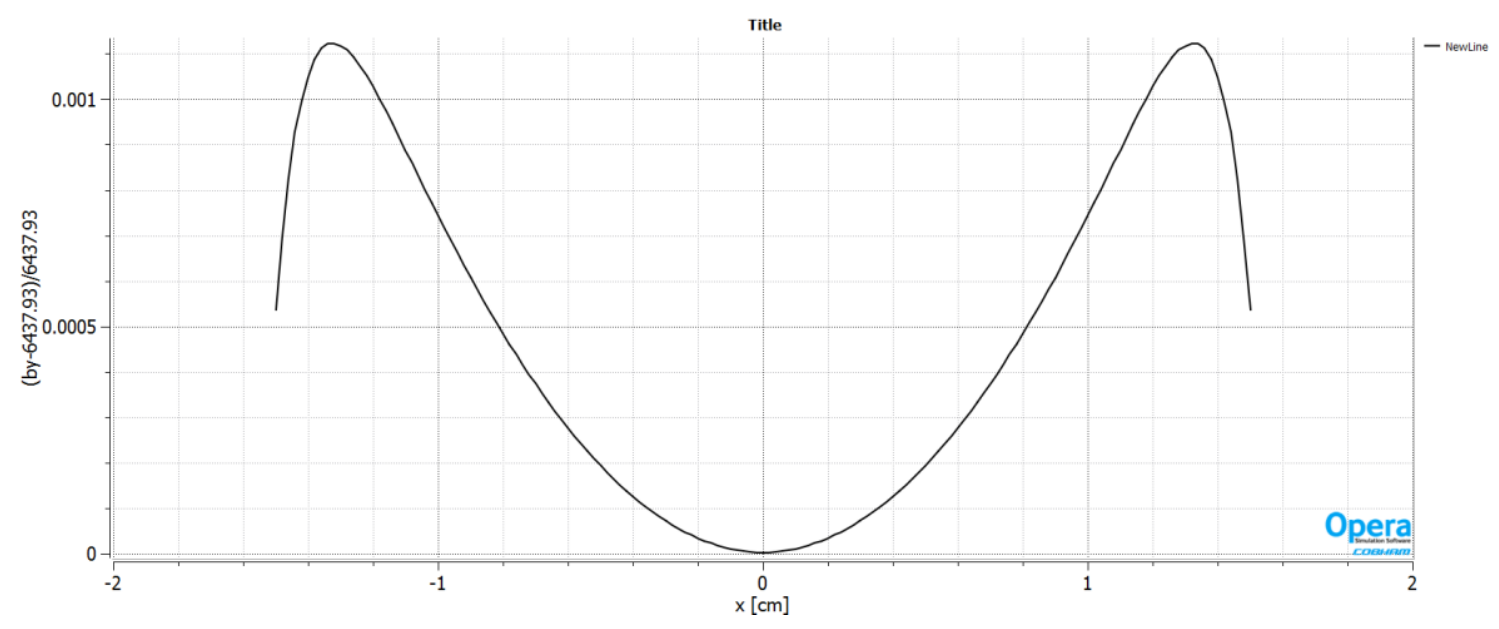

Figure 13. Field homogeneity halfway of the Halbach magnet.

The fringe field of the Halbach magnet along the trajectory of the $144 \mathrm{Mev}$ beam which is shown in Figure 14 can be minimized by either, using magnetic material around the Halbach magnet or using a vacuum pipe for the $144 \mathrm{MeV}$ beam or both.

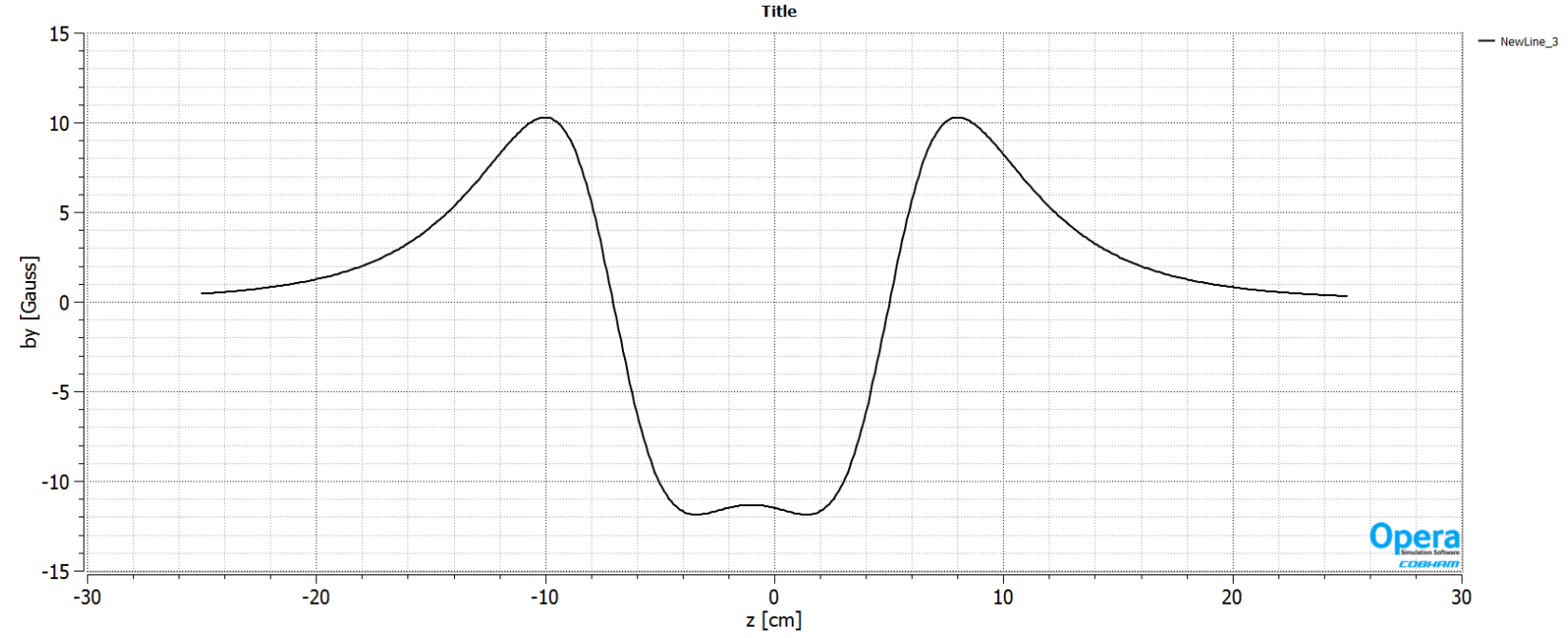

Figure 14. The magnetic field generated by the Halbach magnet along the $144 \mathrm{MeV}$ trajectory. No magnetic shield has been use at either around the Halbach magnet or around the vacuum pipe.

Although the Halbach magnet is less expensive to make and does not require the expensive power supply of the electromagnet, the Halbach magnet is prone to radiation damage from the $150 \mathrm{MeV}$ electrons. To minimize the effect of the radiation on the permanent magnet, $\mathrm{Pb}$ material of $\sim 1.5 \mathrm{~cm}$ thick [3] can be placed in front of the permanent magnet material. Although 1.5 to $2 \mathrm{~cm}$ thick $\mathrm{Pb}$ material can stop the 150 $\mathrm{MeV}$ electron beam the generation of neutrons from the interaction of the electrons with the $\mathrm{Pb}[4]$ is the most damaging effect of the permanent magnet material. 
To mitigate the effect of the radiation damage was proposed [5] to remove the permanent magnet wedges from the horizontal plane and substitute them with material which is not affected by the radiation.This modification of the Halbach magnet is the subject of the study of the next section.

\section{The 3D electromagnetic design of a modified $12 \mathrm{~cm}$ long Halbach type magnet}

Although a Halbach dipole magnet as described earlier seems ideal to be used instead of an electromagnet, there are some objections in using such a magnet in an environment that is subject to radiation. To mitigate the effect of the radiation on the permanent magnet material was suggested [5] to remove the permanent magnet wedges from the horizontal plane and design the magnet to provide a dipole field with acceptable homogeneity. An isometric view of such a modified dipole Halbach magnet is shown in Fig. 15 with the permanent magnet wedges on the horizontal plane removed and replaced by magnetic iron wedges. The holder of the permanent magnet wedges is made of magnetic material to minimize the magnetic field at the region of the $144 \mathrm{MeV}$ beam. The vacuum pipe of the $144 \mathrm{MeV}$ beam is made of magnetic material and is copper plated inside.

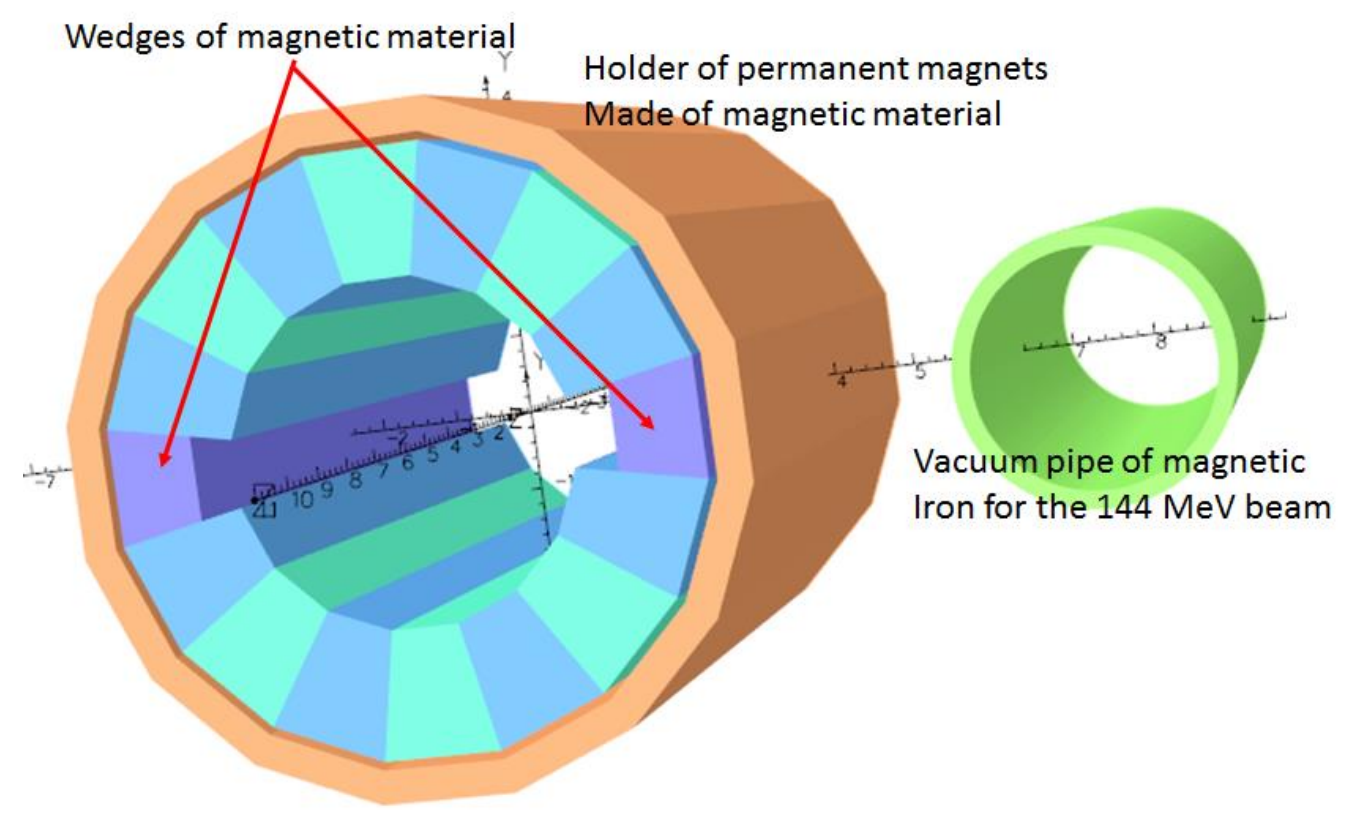

Figure 15. Isometric view of the modified Halbach magnet. The permanent magnet wedges on the horizontal plane have been removed and replaced by magnetic iron wedges. The ring which surrounds the permanent magnet wedges is the holder of the permanent magnet wedges and is made of magnetic iron. The vacuum pipe of the $144 \mathrm{MeV}$ beam is made of magnetic iron and is copper plated inside.

The distance between the center of the Halbach magnet and the center of the vacuum pipe of the 144 $\mathrm{MeV}$ beam at the entrance of the magnet is $6.26 \mathrm{~cm}$. There is some space between the vacuum pipe of the $144 \mathrm{MeV}$ beam and the ring-holder of the permanent magnet wedges for material to serves as cooling conductor to keep the temperature of the permanent magnet material constant. Table 4 lists some of the specifications of the Halbach magnet. The permanent magnet material is NdFeB_N35EH with a remnant field of 12040 Gauss and coercive field of -11700 Oe. 
Table 4. The S4.BEN01 modified Halbach-type magnet specifications

\begin{tabular}{|c|c|c|c|c|c|c|c|}
\hline $\begin{array}{c}\text { Length } \\
{[\mathrm{m}]}\end{array}$ & $\begin{array}{c}\mathrm{B}_{\text {field }} \\
{[\mathrm{T}]}\end{array}$ & $\begin{array}{c}\text { Sagitta } \\
{[\mathrm{cm}]}\end{array}$ & $\begin{array}{c}\mathrm{R}_{\text {in }} \\
{[\mathrm{cm}]}\end{array}$ & $\begin{array}{c}\mathrm{R}_{\text {in }} \\
{[\mathrm{cm}]}\end{array}$ & PM Material & $\begin{array}{c}\mathrm{B}_{\mathrm{r}} \\
{[\mathrm{Gauss}]}\end{array}$ & $\begin{array}{c}\mathrm{H}_{\mathrm{c}} \\
{[\mathrm{Oe}]}\end{array}$ \\
\hline 0.12 & 0.30 & 0.12 & 2.0 & 3.5 & NdFeB_N35EH & 12040 & -11700 \\
\hline
\end{tabular}

The removal of the permanent magnet wedges affects adversely the homogeneity of the Halbach magnet's field. To restore the homogeneity of the field we partly refill the empty spaces with magnetic iron wedges. In addition we also change the direction of the easy axis of the rest of the wedges. Table 5 list the magnetization direction of the easy axis of the wedges for a regular dipole Halbach magnet and a modified one shown on Fig. 15.

Table 5. The magnetization direction of the easy axis of the wedges for a regular Halbach magnet and a modified one.

\begin{tabular}{|c|c|c|c|c|c|c|c|c|c|}
\hline WedgeAngle=> & $0^{\circ}$ & $22.5^{\circ}$ & $45^{\circ}$ & $67.5^{\circ}$ & $90^{\circ}$ & $112.5^{\circ}$ & $135^{\circ}$ & $157.5^{\circ}$ & $180^{\circ}$ \\
\hline Regular & $270^{0}$ & $315^{\circ}$ & $0^{\circ}$ & $45^{\circ}$ & $90^{\circ}$ & $135^{\circ}$ & $180^{0}$ & $225^{\circ}$ & $270^{\circ}$ \\
\hline Modified & NA & $342^{\circ}$ & $3^{\circ}$ & $45^{\circ}$ & $90^{\circ}$ & $135^{\circ}$ & $177^{0}$ & $198^{\circ}$ & NA \\
\hline & & & & & & & & & \\
\hline WedgeAngle=> & & $-157.5^{\circ}$ & $-135^{\circ}$ & $-112.5^{\circ}$ & $-90^{\circ}$ & $-67.5^{\circ}$ & $-45^{\circ}$ & $-22.5^{\circ}$ & \\
\hline Regular & & $315^{\circ}$ & $0^{\circ}$ & $45^{\circ}$ & $90^{\circ}$ & $135^{\circ}$ & $180^{\circ}$ & $225^{\circ}$ & \\
\hline Modified & NA & $342^{\circ}$ & $3^{\circ}$ & $45^{\circ}$ & $90^{\circ}$ & $135^{\circ}$ & $177^{0}$ & $198^{\circ}$ & NA \\
\hline
\end{tabular}

The direction of the magnetization axis of each of the wedges of the modified Halbach magnet as it appears in Table 5 generates a field homogeneity which is shown in Fig.16. The red trace shows that the field homogeneity of the modified Halbach magnet over the beam is better than $10^{-3}$. The center of the 1.2 $\mathrm{cm}$ radius circles in Fig. 16 represents the reference particle of the $150 \mathrm{MeV}$ bunch in the magnet. Further optimization of the modified Halbach magnet may further improve the field homogeneity.

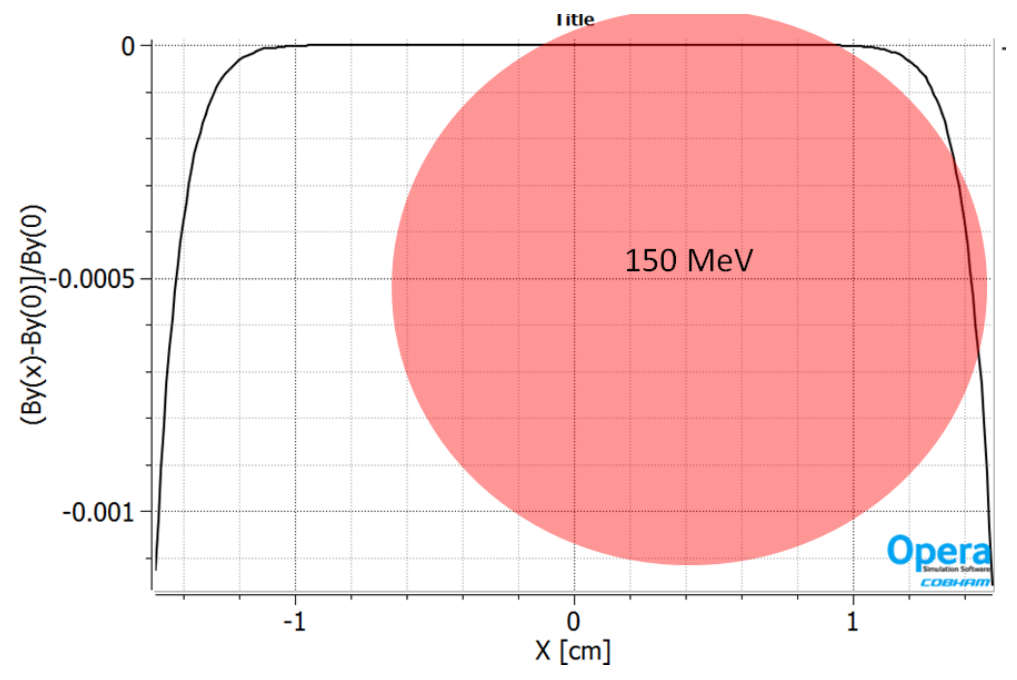

Figure 16. The field homogeneity of the modified Halbach magnet. The center of the $1.2 \mathrm{~cm}$ radius circle is the reference particle of the $150 \mathrm{MeV}$ bunch which is inside the modified Halbach magnet. 
Since the magnetic field generated by the Halbach magnet is constant we have placed a window frame magnet downstream of the S4.BEN01 Halbach magnet to adjust the magnetic field by $\sim \pm 5 \%$. An isometric view of such a magnet model which combines the Halbach magnet with a window frame magnet is shown in Fig. 17.
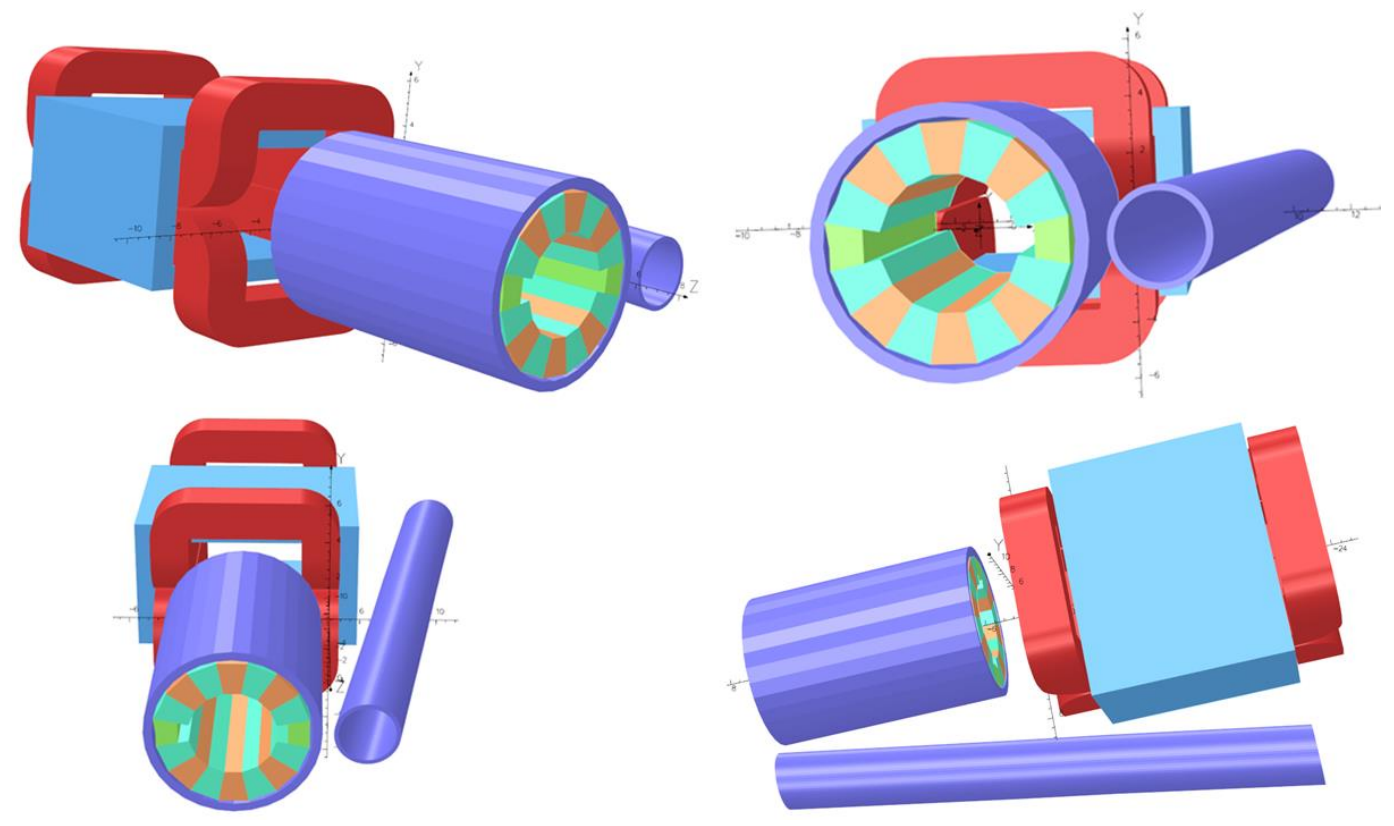

Figure 17. Isometric views of the Habach magnet in combination with a window frame magnet.

\section{Conclusion}

The $20 \mathrm{~cm}$ electromagnet provides variable magnetic field with good homogeneity but for high cost of the power supply. The $12 \mathrm{~cm}$ long Halbach type permanent magnet is smaller in size easier and less expensive to manufacture. It does not require a power supply, and its field homogeneity is complable to that of the electromagnet. A $10 \mathrm{~cm}$ long low field electromagnet can be used to provide adjustability of the $150 \mathrm{MeV}$ beam trajectory. A modified Halbach magnet can mitigate the radiation effect on the permanent magnet material, and also provide a dipole field with good homogeneity comparable to that of the electromagnet.

\section{References}

1 C. Mayes "The CBETA project" arXiv.org/abs/1504.00588 (2015)

${ }^{2} \mathrm{~K}$. Halbach, "Design of permanent multipole magnets with oriented rare earth cobalt material", NIM

169 (1980) pp. 1-10 http://dx.doi.org/10.1016/0029-554X(80)90094-4

${ }^{3}$ http://holbert.faculty.asu.edu/eee460/IonizationRange.pdf

${ }^{4}$ http://www-pub.iaea.org/MTCD/publications/PDF/trs188_web.pdf

${ }^{5}$ Thomas Roser, Private communication 


\section{Acknowledgements}

The authors want to thank the CBETA group that provided some of the information that is used in this study. 